\title{
Cognitive-behavioural therapy in medication-treated adults with attention-deficit/hyperactivity disorder and co-morbid psychopathology: a randomized controlled trial using multi-level analysis
}

\author{
S. Young 1,2,3*, M. Khondoker ${ }^{4,5}$, B. Emilsson ${ }^{4,6}$, J. F. Sigurdsson ${ }^{3,6,7}$, F. Philipp-Wiegmann ${ }^{2,4}$, \\ G. Baldursson ${ }^{6}$, H. Olafsdottir ${ }^{6}$ and G. Gudjonsson ${ }^{2,3,4}$ \\ ${ }^{1}$ Division of Brain Sciences, Department of Medicine, Centre for Mental Health, Imperial College London, London, UK \\ ${ }^{2}$ Broadmoor Hospital, West London Mental Health NHS Trust, Crowthorne, UK \\ ${ }^{3}$ Reykjavik University, Reykjavik, Iceland \\ ${ }^{4}$ King's College London, Institute of Psychiatry, Psychology and Neuroscience, London, UK \\ ${ }^{5}$ Department of Applied Health Research, University College London, London, UK \\ ${ }^{6}$ Landspitali - The National University Hospital of Iceland, Reykjavik, Iceland \\ ${ }^{7}$ University of Iceland, Reykjavik, Iceland
}

\begin{abstract}
Background. Attention-deficit/hyperactivity disorder (ADHD) is a neurodevelopmental disorder characterized by high rates of co-morbid psychopathology. Randomized controlled trials of multimodal interventions, combining pharmacological and psychological treatments, have shown a robust treatment effect for ADHD symptoms but outcomes for co-morbid symptoms have been mixed. This may be accounted for by the type of intervention selected and/or by methodological problems including lack of follow-up and low power. The current study addressed these limitations in a parallel-group randomized controlled trial conducted in Iceland.
\end{abstract}

\begin{abstract}
Method. A total of 95 adult ADHD patients who were already being treated with medication (MED) were randomly assigned to receive treatment as usual (TAU/MED) or 15 sessions of cognitive-behavioural therapy (CBT/MED) using the RER2ADHD intervention which employs both group and individual modalities. Primary measures of ADHD symptoms and severity of illness, and secondary measures of anxiety, depression and quality of life were given at baseline, end of treatment and 3-month follow-up. Primary outcomes were rated by clinicians blind to treatment condition assignment.

Results. CBT/MED showed overall (combined outcome at end of treatment and 3-month follow-up) significantly greater reduction in primary outcomes for clinician-rated and self-rated ADHD symptoms. Treatment effect of primary outcomes was maintained at follow-up, which suggests robust and lasting findings. In contrast to the primary outcomes, the secondary outcomes showed significant improvement over time.
\end{abstract}

Conclusions. The study provides evidence for the effectiveness of $R \mathcal{E} R 2 A D H D$ and demonstrates that there are differential effects over time for ADHD symptoms versus co-morbid problems, the latter taking longer to show positive effects.

Received 1 December 2014; Revised 23 March 2015; Accepted 1 April 2015; First published online 29 May 2015

Key words: Attention-deficit/hyperactivity disorder, cognitive-behavioural therapy, randomized controlled trials, reasoning and rehabilitation, $R \& R 2$, treatment.

\section{Introduction}

Attention-deficit/hyperactivity disorder (ADHD) is a common neurodevelopmental disorder characterized by core symptoms of inattention, hyperactivity and/or impulsivity and associated with significant social, educational and occupational impairments. Longitudinal

* Address for correspondence: S. Young, Division of Brain Sciences, Department of Medicine, Centre for Mental Health, Imperial College London, 37 Claybrook Road, London W6 8LN, UK.

(Email: susan.young1@imperial.ac.uk) and epidemiological studies have shown ADHD to be a chronic life-long condition emerging in childhood (Biederman \& Faraone, 2005; Sobanski et al. 2007; Young \& Amarasinghe, 2010; Guldberg-Kjär et al. 2013). Meta-analytic reviews have estimated the prevalence of ADHD in adulthood to be $2.5-5 \%$ (Simon et al. 2009; Willcutt, 2012). It is a complex disorder characterized by high rates of co-morbidity including mood disorders, anxiety, alcohol and drug abuse, and interpersonal relationship problems (Pliszka, 1998; Shaw et al. 2012). Indeed cross-sectional, retrospective and follow-up studies have indicated that ADHD patients

This is an Open Access article, distributed under the terms of the Creative Commons Attribution licence (http://creativecommons. org/licenses/by/3.0/), which permits unrestricted re-use, distribution, and reproduction in any medium, provided the original work is properly cited. 
are up to $80 \%$ more likely to develop other psychiatric difficulties (Sobanski et al. 2007; Barkley et al. 2008). A large, nationally representative US sample demonstrated that ADHD had an increased lifetime prevalence of all psychiatric disorders, even after adjusting for sociodemographic characteristics (Bernardi et al. 2012).

In the UK, pharmacological interventions are the first-line recommended treatment of ADHD in adults (National Institute for Health and Clinical Excellence, 2009; Bolea-Alamañac et al. 2014) and meta-analytic studies have reported a medium to large treatment effect (Mészáros et al. 2009). However, up to $50 \%$ of medicated adults may not fully respond to medication (Wender, 1998; Wilens et al. 2002; Prince, 2006; Safren, 2006). By contrast, psychological treatments have received less attention but generally support that medication significantly augments the outcome of therapy; a systematic review examining the effect of treatment modality on long-term outcomes concluded that the combination of pharmacological and non-pharmacological treatment was most consistently associated with improved longterm outcomes and large effect sizes. Furthermore, the age of treatment initiation and duration of treatment did not markedly affect the proportion of improved outcomes reported (Shaw et al. 2012). Only one study has been conducted comparing participants randomized to receive combined cognitive-behavioural therapy (CBT) and medication (dextroamphetamine) versus CBT and placebo; this study found ADHD symptom outcomes using CBT alone were as good as when both medication and CBT interventions were combined (Weiss et al. 2012). These findings are impressive and have great implications for treatment with CBT as a stand-alone intervention in this population. However the findings need to be replicated.

International guidelines recommend a multimodal treatment approach comprising both pharmacological and psychological interventions (Seixas et al. 2012). The common clinical reality, however, is a reliance on medication as first line. This is due to the limited evidence base currently available for psychological intervention for treating adults with ADHD. More research is needed in this area and this study makes an important contribution to the field. When choosing psychological interventions, the selection of groupdelivered treatment is preferable as it is resource and cost effective (National Institute for Health and Clinical Excellence, 2009). Randomized controlled trials (RCTs) that evaluate the combination of these treatments (versus medication alone) have reported medium to large treatment effects for both self-rated and clinician-rated improvements in ADHD symptoms (Stevenson et al. 2002; Safren et al. 2005, 2010; Safren, 2006). Furthermore, some treatment effects appear to be robust, with two studies reporting sustained effects 1 year later (Stevenson et al. 2002; Safren et al. 2010).

There have only been three RCTs involving a group psychological intervention that have evaluated comorbid psychopathology at outcome (i.e. anxiety and depression). These have reported mixed findings; in contrast to the findings of Emilsson et al. (2011), two studies reported no significant improvement in symptoms (Solanto et al. 2010; Hirvikoski et al. 2011). The weakness with the latter two studies is that there were no follow-up data, which means that any possible reduction in co-morbid symptoms after the end of treatment was not assessed. Emilsson et al. (2011) compared medicated patients randomly assigned to either receive medication (MED) and CBT (CBT/MED) or treatment as usual (TAU/MED) and reported large treatment effects at 3-month follow-up for self-rated attention, hyperactivity/impulsivity, anxiety, depression, emotional control, antisocial behaviour and social functioning and clinician ratings of illness severity and ADHD core symptoms. However, there was no significant treatment effect for anxiety and depression at the end of treatment, which is consistent with the Hirvikoski et al. (2011) and Solanto et al. (2010) studies, and suggests that co-morbid problems may take longer to show positive effect than the core ADHD symptoms. This finding requires further investigation.

However, the Emilsson et al. (2011) community study only involved 54 participants, 27 in each group, and there was a substantial amount of missing data at the end of treatment and at 3-month follow-up for both the CBT/MED and TAU/MED groups. The presence of missing data may potentially lead to a biased estimate of the treatment effect, which was not appropriately dealt with in the previous study due to the limitations of the statistical approach taken. For example, there is evidence that age and antisocial personality (ASP) traits are significant predictors of failure to attend follow-up appointments (Gudjonsson et al. 2004). A recommended way (White et al. 2011) to reduce possible bias is to analyse all the observed outcome data via the maximum likelihood method under the data missing at random (MAR) assumption, which requires the inclusion of any relevant predictors of missing data in the analysis model.

The current study therefore addressed these methodological weaknesses by using a larger sample randomized to either CBT/MED or TAU/MED and including a 3-month follow-up. We performed an intention-to treat analysis using a linear mixed model and analysed for three possible predictors of missing data: gender, age, and ASP traits. Thus we analysed the effects of treatment over time (i.e. end of treatment versus at 3-month follow-up) as well as overall group differences in the outcome measures whilst controlling for possible group imbalances caused by missing data. It 
was hypothesized that the CBT/MED group would show significantly greater reduction in the primary outcomes of ADHD core symptoms and illness severity compared with the TAU/MED group after adjusting for missing data and possible confounders. A similar treatment effect was expected for secondary outcomes of anxiety, depression and quality of life. Treatment gains were expected to be maintained at 3-month follow-up.

\section{Method}

\section{Trial design}

A parallel-group RCT was conducted at an ADHD outpatient setting within the Mental Health Services at Landspitali - The National University Hospital of Iceland. All participants meeting inclusion criteria were independently and individually randomly allocated (1:1) to receive the RER2ADHD programme (CBT/MED) or treatment as usual (TAU/MED). Assessments occurred at three time points: baseline, end of treatment and 3 months after treatment. The study was registered with the International Clinical Trials Registry (no. 12611000533998).

An a priori power calculation was conducted using $G^{*}$ Power (Faul et al. 2007) to estimate the sample size required. Safren et al. (2005) obtained a large effect size between CBT-treated medicated adults with ADHD and those treated by medication only. In view of the fact that the Safren et al. study involved individual rather than group therapy, where the effect size is likely to be larger, we used an estimated effect size of 0.50 . The power was set at $80 \%$ and the level of significance at 0.05 . This suggested a sample of $n=51$ in each group.

\section{Participants}

Participants were either hospital referrals for outpatient rehabilitation made by the Mental Health Services at the Landspitali University Hospital, referrals from private practitioners or self-referrals from an advertisement placed with a national ADHD support group (Icelandic ADHD Association). Participants were eligible for the study if they were over 18 years of age, had a current ADHD diagnosis and had been stable on prescribed ADHD medication for at least 1 month. Participants were asked to keep dosages unchanged during the study. Exclusion criteria were severe mental illness (i.e. psychotic disorders, bipolar disorder), severe eating disorder, active suicidal ideation, active drug abuse and history of intellectual impairment as the treatment programme would not be suitable for these patient groups without modification. Exclusion criteria were assessed from a review of medical records, in addition to a baseline assessment by an experienced mental health practitioner (see Baseline assessments section).

Of 187 referrals, 73 (39\%) were received from private practice psychiatrists, 56 (30\%) were referred by psychiatrists, psychologist or psychiatric nurses at the Mental Health Services at Landspitali University Hospital and $56(30 \%)$ were referred from advertisements to the members of the Icelandic ADHD Association. Out of the 187 referrals, 95 (51\%) participants took part in the study. Fig. 1 presents the reasons for non-participation, the most common being declining to participate $(n=30)$, not contactable $(n=18)$ and stopped medication $(n=16)$. Of the participants, 11 were excluded because at the study intake interview (conducted by an experienced mental health practitioner) they were not diagnosed with ADHD [according to Diagnostic and Statistical Manual of Mental Disorders (DSM)-IV criteria]; 62 of the participants were female (mean age $=35.00$, S.D. $=11.81$ years) and 33 were male (mean age $=$ 35.45 , S.D. $=11.62$ years).

Demographic and clinical characteristics of the study sample are presented in Table 1. All medication was prescribed by psychiatrists and, at baseline, 79 (83.2\%) were taking methylphenidate and $16(16.8 \%)$ atomoxetine. Five participants were also taking bupropion. In addition, $63(66.3 \%)$ participants were taking other prescribed medications (mean number of medications 2.45, s.D. $=$ 1.39) including antidepressants, benzodiazepines, insulin and ibuprofen. There was considerable co-morbidity present as, in addition to ADHD, participants reported comorbid depression $(63.2 \%)$, anxiety $(36.8 \%)$, and history of drug/alcohol abuse (15.8\%). Of the participants, seven $(7.4 \%)$ reported to have been diagnosed with a personality disorder and four with Asperger's syndrome in childhood. Four reported having post-traumatic stress disorder and two with a history of eating disorder.

\section{Interventions}

$R \mathcal{E} R 2 A D H D$ is a CBT intervention programme developed for youth and adults with ADHD (Young \& Ross, 2007). It is a revised version of the 35-session Reasoning \& Rehabilitation prosocial competence training programme which has a strong evidence base (Tong \& Farrington, 2006). It was revised to be a shorter and more relevant intervention for individuals presenting with symptoms associated with ADHD. The revision, RER $2 A D H D$, is a structured, manualized programme consisting of 15 sessions of $90 \mathrm{~min}$ (excluding a mid-session break) and aims to decrease ADHD symptoms and improve social, problem-solving and organizational skills. It has five treatment modules: (a) neurocognitive, e.g. learning strategies to improve attentional control, memory, 


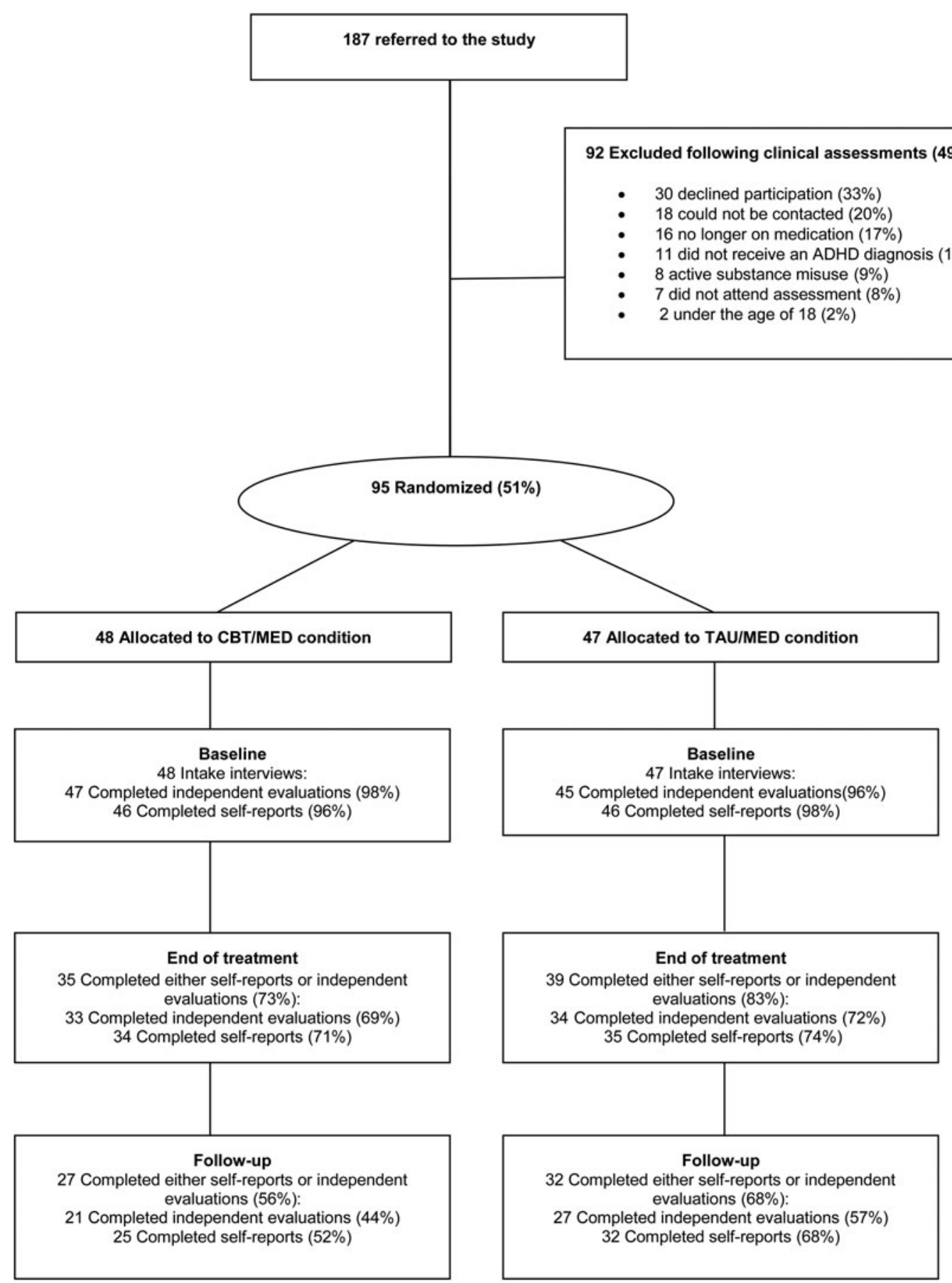

Fig. 1. Flowchart of patient participation. ADHD, Attention-deficit/hyperactivity disorder; CBT/MED, cognitive-behavioural therapy plus medication; TAU/MED, treatment as usual plus medication.

impulse control and planning; (b) problem solving, e.g. developing skilled thinking, problem identification, consequential thinking, managing conflict and making choices; (c) emotional control, e.g. managing feelings of anger and anxiety; (d) prosocial skills, e.g. recognition of the thoughts and feelings of others, empathy, negotiation skills, and conflict resolution; and (e) critical reasoning, e.g. evaluating options and effective behavioural skills. RER2ADHD is a group treatment supplemented by one-to-one meetings with a mentor. In the present study the group sessions were delivered twice per week. The mentors met with the participants between each group session in order to support participants to transfer skills learned in the group into their daily lives. Programme integrity was ensured by group sessions being delivered according to the manual by 
Table 1. Demographic, clinical and baseline characteristics of the study sample $(n=95)$

\begin{tabular}{|c|c|c|c|c|}
\hline & $\begin{array}{l}\text { Total } \\
(n=95)\end{array}$ & $\begin{array}{l}\text { CBT/MED } \\
(n=48)\end{array}$ & $\begin{array}{l}\text { TAU/MED } \\
(n=47)\end{array}$ & Statistics \\
\hline \multicolumn{5}{|l|}{ Gender, $n(\%)$} \\
\hline Men & $33(43.7)$ & $18(37.5)$ & $15(31.9)$ & \multirow[t]{2}{*}{$\chi^{2}=0.327, p=0.57$} \\
\hline Women & $62(65.3)$ & $30(62.5)$ & $32(68.1)$ & \\
\hline \multicolumn{5}{|l|}{ Age, years } \\
\hline Mean & 35.17 & 34.19 & 36.17 & \multirow[t]{3}{*}{$t_{93}=0.826, p=0.41$} \\
\hline S.D. & 11.68 & 10.58 & 12.75 & \\
\hline Range & $18-73$ & $18-68$ & $18-73$ & \\
\hline \multicolumn{5}{|l|}{ Marital status, $n(\%)$} \\
\hline Single & $47(49.5)$ & $23(48.9)$ & $24(51.1)$ & \multirow[t]{2}{*}{$\chi^{2}=0.189, p=0.66$} \\
\hline In a relationship & $47(49.5)$ & $24(51.1)$ & $23(48.9)$ & \\
\hline \multicolumn{5}{|l|}{ Employment status, $n(\%)$} \\
\hline Employed & $41(43.2)$ & $18(37.5)$ & $23(48.9)$ & \multirow[t]{3}{*}{$\chi^{2}=2.236, p=0.33$} \\
\hline Training & $22(23.2)$ & $14(29.2)$ & $8(17.0)$ & \\
\hline Pension/unemployed & $32(33.7)$ & $16(33.3)$ & $16(34.0)$ & \\
\hline \multicolumn{5}{|l|}{ Medical history, $n(\%)$} \\
\hline History of serious illness & $27(28.4)$ & $13(27.1)$ & $14(29.8)$ & \multirow{4}{*}{$\begin{array}{l}\chi^{2}=0.085, p=0.77 \\
\chi^{2}=0.118, p=0.73 \\
\chi^{2}=0.020, p=0.89 \\
\chi^{2}=0.735, p=0.39\end{array}$} \\
\hline History of head trauma & $36(37.9)$ & 19 (39.6) & $17(36.2)$ & \\
\hline History of serious accidents & $33(34.7)$ & $17(35.4)$ & $16(34.0)$ & \\
\hline History of receiving psychotherapy & $69(72.6)$ & $33(68.8)$ & $36(76.6)$ & \\
\hline \multicolumn{5}{|l|}{ ADHD-specific medication, $n(\%)$} \\
\hline Methylphenidate & $73(83.2)$ & $40(83.3)$ & $33(83.0)$ & \multirow{4}{*}{$\begin{array}{l}\chi^{2}=0.002, p=0.963 \\
\chi^{2}=0.002, p=0.963 \\
\chi^{2}=0.234, p=0.629 \\
\chi^{2}=0.005, p=0.942\end{array}$} \\
\hline Atomoxetine & $16(16.8)$ & $8(16.7)$ & $8(17.0)$ & \\
\hline Bupropion & $5(5.3)$ & $3(6.3)$ & $2(4.3)$ & \\
\hline Other medications $^{\mathrm{a}}$ & $63(66.3)$ & $32(66.7)$ & $31(66.0)$ & \\
\hline
\end{tabular}

CBT/MED, Cognitive-behavioural therapy plus medication; TAU/MED, treatment as usual plus medication; s.D., standard deviation; ADHD, attention-deficit/hyperactivity disorder.

${ }^{a}$ Other medications include, for example, antidepressants, benzodiazepines, insulin, ibuprofen and various other medications.

experienced CBT therapists, who had received training and accreditation to deliver the programme. The mentoring sessions were provided by psychology students who also received training, supervision and written guidance.

Treatment completion was classified as $\geq 12$ sessions, representing $80 \%$ attendance of the programme.

TAU was classified as receiving usual treatment, which included both pharmacological and nonpharmacological treatments.

\section{Measures}

Baseline assessments

All referrals who could be contacted and who consented to participate in the study were interviewed prior to randomization to ascertain clinical diagnosis according to DSM-IV criteria using the MINI International Neuropsychiatric Interview (Sheehan et al. 1998) by an experienced mental health practitioner. In addition, the 54-item Gough Socialization Scale (Gough, 1960) was used to measure ASP traits because these have been found to be associated with failure to attend follow-up appointments after treatment in an Icelandic sample (Gudjonsson et al. 2004). In addition, Young \& Gudjonsson (2006) found that community patients diagnosed with ADHD commonly had ASP traits as measured by the Gough Socialization Scale, which might be related to missing follow-up data (dropouts). There may of course be other possible confounding variables not included in the analysis, such as substance misuse, which may act similarly to APD traits.

Sociodemographic data and medical information from a review of clinical records were obtained (see Table 1). In addition, a battery of psychometric tests assessed the primary (ADHD core symptoms and illness severity) and secondary outcomes (anxiety, depression, quality of life) at baseline, at the end of treatment and at 3-month follow-up as follows.

\section{Primary outcomes}

The Kiddie-Schedule for Affective Disorders and Schizophrenia (K-SADS), ADHD section, is a measure 
of symptom change and severity of ADHD symptomatology (Kaufman et al. 1996). The 18-item questionnaire was completed by clinicians who were blind to treatment allocation. Magnússon et al. (2006) have reported that the scale has good reliability and validity in an Icelandic sample.

The Clinical Global Impression (CGI; National Institute of Mental Health, 1985) is a single-question observer rating of severity of illness on a seven-point scale. It is based on judgment regarding impairment in functioning, symptom severity and distress or coping and is supported by examples of these factors. It was completed by clinicians who were blind to treatment condition. The CGI has been widely used in treatment evaluation studies and has been found to correlate with ADHD severity measured by the adult ADHD Investigator Symptom Rating Scale (Spencer et al. 2010).

The Barkley Current Symptoms Scale (Barkley, 1998) is an 18-item self-report questionnaire that measures ADHD symptoms and corresponds with the DSM-IV criteria (APA, 1994). The questionnaire consists of a total scale made up from two subscales; one measures inattention and the other hyperactivity/impulsivity. Magnússon et al. (2006) found a high correlation between informants' ratings of symptoms and interviewbased diagnoses in childhood and adulthood in an Icelandic sample. The correlations were 0.49 (males) and 0.58 (females) for childhood symptoms and 0.50 (males) and 0.55 (females) for symptoms in adulthood.

\section{Secondary outcomes}

The Beck Anxiety Inventory (BAI; Beck \& Steer, 1993) is a 21-item self-report questionnaire that is widely used in clinical practice and research.

The Beck Depression Inventory (BDI; Beck et al. 1961) is a 21-item self-report questionnaire to assess symptoms and severity of depression.

The Quality of Life Scale (QOLS; Flanagan, 1978, 1982) is a 16-item scale that assesses attitudes toward a person's own quality of life. The QOLS was originally developed for research in healthy populations, but it has been used in several international studies with chronic illnesses (Burckhardt \& Anderson, 2003) and among cancer patients in Iceland (Friðriksdóttir et al. 2011). Burckhardt \& Anderson (2003) have demonstrated good construct validity of the scale in a sample of chronically ill and healthy adults from American and Swedish databases.

\section{Procedure}

All referrals who could be contacted and who agreed to participate in the study were assessed for eligibility by participating in an interview to confirm their ADHD diagnosis, and assess co-morbidity. A battery of self-rated and clinician-rated evaluations (the latter being blind to treatment allocation) was conducted at the three time points (baseline, post-treatment and at 3-month followup). Participants were randomized to either the CBT/ MED or TAU/MED condition. Randomization was conducted independently by a psychiatrist at Landspitali University Hospital, who was not involved in the study. The psychiatrist had no information about the participants and received numbers that were preassigned to the participants. Block randomization by using equal block sizes was performed at the time of each study phase. Only the final randomization numbers were reported back to the researchers to protect the concealment of the allocation as proposed in various studies (Beller et al. 2002; Schulz \& Grimes, 2002). The $R \mathcal{E} 2 A D H D$ programme was delivered twice per week by experienced CBT therapists who had attended training to deliver the programme. Group participants met their mentor between group sessions for at least $30 \mathrm{~min}$. Mentors attended a training session to fulfil this role involving an introduction to the programme and the mentoring role. In addition, mentors have a manual that guides them through topics to be discussed within the mentoring session. They received supervision once a fortnight from the lead group therapist. There were five RER2ADHD treatment groups in total. Participants in the TAU/MED condition received pharmacological intervention and other non-pharmacological interventions but these were not systematically provided or recorded.

\section{Statistical analyses}

The statistical analysis involved two sequential steps. First, we applied a logistic regression model to identify factors (i.e. gender, age and ASP traits) that might predict the probability of missing data. Only age was found to be significantly associated with the probability of dropouts (i.e. younger participants in both groups more often failed to attend the assessment interviews with the independent raters: K-SADS, $Z=-2.19, p=0.029$; CGI, $Z=-2.35, p=0.019$ ).

Second, an intention-to-treat analysis (individuals analysed in the group to which they were randomized) of available outcome data was performed to estimate the effect of offering the treatment using a linear mixed model. In view of the significant relationship found between age and missing data dropouts, we controlled for age in the linear mixed model. The random component of the mixed model included a random intercept term for subject identifier to take account of between-subject variability and the correlation between the repeated measures. Within the fixed part of the model, the treatment effect was adjusted for time (a binary indicator of whether an 
outcome measure corresponds to follow-up or end of treatment) and the baseline measures of the respective outcome in all models. We tested condition $\times$ time interactions, but none was found statistically significant and therefore these were excluded from the model.

The amount of missing data at baseline was minimal, but there was a substantial proportion (leading up to $50 \%$ by the follow-up) of missing data in the completion of the outcome measures due to study dropouts. The dropout rate was similar for both the CBT/MED and TAU/MED groups (see Fig. 1), with $\chi^{2}$ tests revealing no differences between groups.

Two of the commonly recommended approaches for dealing with the risk of potential bias due to missing data are multiple imputation and complete case analysis via maximum likelihood. Multiple imputation is appropriate when there are missing data in covariates which is not the case in our study. When missing data occur only in the outcome variables then complete case analysis via maximum likelihood produces unbiased results, provided that the variables associated with the outcome being missing are included as covariates (under a MAR assumption). We adopted the complete case analysis approach under a MAR assumption as we had missing data only in the outcome variables. Covariates driving missingness were identified using a logistic regression analysis and an analysis of all observed outcome data was performed using a linear mixed model via maximum likelihood method controlling for predictors of missing data, which should produce unbiased estimates under a MAR assumption (White et al. 2011).

Adjusted effect sizes (Cohen's $d$ ) were obtained by calculating the residuals from the respective linear mixed model with the condition term excluded, and then calculating the standardized mean difference of the adjusted outcome (residuals) between groups. The calculation was conducted using the user contributed STATA module COHEND (http://ideas.repec.org/ c/boc/bocode/s457235.html), which calculates effect sizes by adjusting for uneven group sizes.

Descriptive characteristics of the demographic and clinical sample data as well as the outcome measures are presented as means and standard deviations. To assess differences between the CBT/MED and TAU/MED conditions at baseline, independent-sample $t$ tests were performed and $\chi^{2}$ tests were used to analyse categories and categorical data, respectively.

\section{Ethical standards}

All procedures contributing to this work comply with the ethical standards of the relevant national and institutional committees on human experimentation and with the Helsinki Declaration of 1975, as revised in 2008.

\section{Results}

\section{Baseline characteristics}

There were no significant differences between the CBT/ MED and the TAU/MED groups in the demographic background data (see Table 1). As far as the baseline outcome measures are concerned, there were no significant differences between groups with the exception that the TAU group had a lower Barkley Current Symptoms Scale hyperactivity/impulsivity score compared with the CBT group (Table 2).

\section{Programme completion}

Just over half of the participants completed the programme $(n=25 ; 52.1 \%)$.

\section{Outcomes}

In Table 3 a selected output from the linear mixed model analyses is given. Each row shows the coefficient of the treatment indicator $(0=$ TAU/MED, $1=$ CBT/MED) and the relevant inferential statistics of the named outcome. All models included a random intercept term for subject identification and controlled for age, time (indicator of whether the measurement corresponds to end of treatment or follow-up or end of treatment) and the baseline measurement differences of each respective outcome variable. Estimates of the adjusted overall mean differences (i.e. combining the scores from end of treatment and at 3-month follow-up) between the CBT/MED and TAU/MED groups and the corresponding $p$ values are provided. There was a significant main effect for all the outcome measures except for the BAI and quality of life measures. The group difference on the BAI was very close to being statistically significant at the 5\% level, which suggests that the CBT group tended to have a considerably reduced BAI scores compared with the TAU group.

There was an overall effect of time (end of treatment versus 3-month follow-up) adjusted for baseline, group and age, on the three secondary measures: BAI $(Z=-2.53$, $p=0.011, d=0.58)$, BDI $(Z=-2.2, p=0.025, d=0.52)$ and quality of life $(Z=2.47, p=0.014, d=0.56)$, showing steady improvement over time in the treatment group. No significant time change was noted regarding the primary outcome measures.

\section{Discussion}

This was a randomized controlled (intention-to-treat analysis) study of the effectiveness of CBT (RER2ADHD) in medication-treated adults with ADHD. The study employed a sophisticated analysis (using a linear mixed model) to control for confounders associated with 
Table 2. Outcome measures in the CBT/MED and TAU/MED conditions and statistics of the baseline measurements

\begin{tabular}{|c|c|c|c|c|c|c|c|}
\hline \multirow[b]{2}{*}{ Outcome } & \multicolumn{3}{|l|}{ CBT/MED } & \multicolumn{3}{|l|}{ TAU/MED } & \multirow{2}{*}{$\begin{array}{l}\text { Statistics of the baseline } \\
\text { measurements between } \\
\text { groups }\end{array}$} \\
\hline & Baseline & End of treatment & Follow-up & Baseline & End of treatment & Follow-up & \\
\hline \multirow{2}{*}{$\begin{array}{l}\text { K-SADS total } \\
n\end{array}$} & $39.96(5.71)$ & $30.60(7.33)$ & $30.95(5.53)$ & $37.53(8.05)$ & $35.47(6.40)$ & $35.67(5.86)$ & $t_{79}=1.253, p=0.214$ \\
\hline & 47 & 33 & 21 & 45 & 34 & 27 & \\
\hline \multirow{2}{*}{$\begin{array}{l}\text { K-SADS inattention } \\
n\end{array}$} & $21.70(3.54)$ & $16.55(3.98)$ & $17.43(3.60)$ & $20.87(4.05)$ & $19.88(3.94)$ & $19.26(3.49)$ & $t_{90}=1.054, p=0.296$ \\
\hline & 47 & 33 & 21 & 45 & 34 & 27 & \\
\hline \multirow{2}{*}{$\begin{array}{l}\text { K-SADS hyperactivity/impulsivity } \\
n\end{array}$} & $17.66(3.76)$ & $14.06(4.45)$ & $13.52(3.86)$ & $16.67(4.78)$ & $15.58(4.15)$ & $16.41(3.93)$ & $t_{90}=1.110 ; p=0.270$ \\
\hline & 47 & 33 & 21 & 45 & 34 & 27 & \\
\hline \multirow{2}{*}{$\begin{array}{c}\text { CGI } \\
n\end{array}$} & $3.96(0.81)$ & $3.03(1.05)$ & $3.14(0.79)$ & $3.91(1.10)$ & $3.79(0.77)$ & $3.80(0.96)$ & $t_{90}=0.231, p=0.818$ \\
\hline & 47 & 33 & 21 & 45 & 34 & 27 & \\
\hline \multirow{2}{*}{$\begin{array}{l}\text { BCS combined } \\
n\end{array}$} & $28.80(9.26)$ & $17.26(7.58)$ & $14.72(8.31)$ & 25.15 (11.09) & $21.57(9.75)$ & $22.34(9.17)$ & $t_{90}=1.714, p=0.090$ \\
\hline & 46 & 34 & 25 & 46 & 35 & 32 & \\
\hline \multirow{2}{*}{$\begin{array}{l}\text { BCS inattention } \\
n\end{array}$} & $16.28(5.49)$ & $10.59(4.40)$ & $9.60(5.34)$ & $15.58(6.57)$ & $13.71(5.72)$ & $14.19(5.85)$ & $t_{90}=0.637, p=0.526$ \\
\hline & 46 & 34 & 25 & 46 & 35 & 32 & \\
\hline \multirow{2}{*}{$\begin{array}{l}\text { BCS hyperactivity/impulsivity } \\
n\end{array}$} & $12.52(5.16)$ & $6.68(5.01)$ & $5.12(4.05)$ & $9.63(5.97)$ & $7.86(5.92)$ & $8.16(5.13)$ & $t_{90}=2.448, p=0.016$ \\
\hline & 46 & 34 & 25 & 46 & 35 & 32 & \\
\hline \multirow{2}{*}{$\begin{array}{l}\text { BAI anxiety } \\
n\end{array}$} & $13.84(8.69)$ & $10.41(9.10)$ & $6.29(4.53)$ & $14.60(7.43)$ & $14.37(10.67)$ & $12.13(7.65)$ & $t_{87}=-0.443, p=0.659$ \\
\hline & 44 & 34 & 24 & 45 & 35 & 31 & \\
\hline \multirow{2}{*}{$\begin{array}{l}\text { BDI depression } \\
n\end{array}$} & $12.72(8.62)$ & $8.38(6.99)$ & $5.04(5.60)$ & $16.09(10.23)$ & $14.00(10.45)$ & $13.14(7.99)$ & $t_{87}=-1.690, p=0.095$ \\
\hline & 46 & 34 & 24 & 43 & 34 & 29 & \\
\hline \multirow{2}{*}{$\begin{array}{l}\text { Quality of life } \\
n\end{array}$} & 71.38 (14.40) & $74.50(14.53)$ & $79.84(11.07)$ & $69.54(14.91)$ & $70.94(16.29)$ & $72.22(14.31)$ & $t_{89}=0.595, p=0.553$ \\
\hline & 45 & 34 & 25 & 46 & 35 & 32 & \\
\hline
\end{tabular}

Data are given as mean (standard deviation).

CBT/MED, Cognitive-behavioural therapy plus medication; TAU/MED, treatment as usual plus medication; K-SADS, Kiddie-Schedule for Affective Disorders and Schizophrenia, attention-deficit/hyperactivity disorder section; CGI, Clinical Global Impression; BCS, Barkley Current Symptoms Scale; BAI, Beck Anxiety Inventory; BDI, Beck Depression Inventory. 
Table 3. Estimated treatment effect from the linear mixed-model analyses with adjusted effect sizes (Cohen's d) from the model

\begin{tabular}{|c|c|c|c|c|c|}
\hline Outcome & $\beta$ Coefficient & S.E. & $p$ & $95 \% \mathrm{CI}$ & Effect size: $d$ \\
\hline K-SADS total & -5.41 & 1.03 & $<0.001$ & -7.43 to -3.38 & 0.65 \\
\hline K-SADS inattention & -3.22 & 0.70 & $<0.001$ & -4.6 to -1.84 & 0.56 \\
\hline K-SADS & -2.11 & 0.60 & $<0.001$ & -3.29 to -0.93 & 0.51 \\
\hline \multicolumn{6}{|l|}{ hyperactivity/impulsivity } \\
\hline CGI & -0.79 & 0.17 & $<0.001$ & -1.12 to -0.46 & 0.64 \\
\hline BCS combined & -6.60 & 1.33 & $<0.001$ & -9.19 to -3.99 & 0.46 \\
\hline BCS inattention & -3.63 & 0.80 & $<0.001$ & -5.21 to -2.06 & 0.42 \\
\hline BCS & -3.10 & 0.73 & $<0.001$ & -4.50 to -1.63 & 0.46 \\
\hline \multicolumn{6}{|l|}{ hyperactivity/impulsivity } \\
\hline BAI anxiety & -3.11 & 1.72 & 0.071 & -6.49 to 0.26 & 0.21 \\
\hline BDI depression & -4.84 & 1.51 & 0.001 & -7.79 to -1.89 & 0.32 \\
\hline Quality of life & 3.36 & 2.50 & 0.180 & -1.55 to 8.26 & 0.14 \\
\hline
\end{tabular}

S.E., Standard error; CI, confidence interval; K-SADS, Kiddie-Schedule for Affective Disorders and Schizophrenia, attentiondeficit/hyperactivity disorder section; CGI, Clinical Global Impression; BCS, Barkley Current Symptoms Scale; BAI, Beck Anxiety Inventory; BDI, Beck Depression Inventory.

missing data, between-subject variability, and the correlation between the measures over time. The current study thus provides a more rigorous methodology and robust statistical analysis of outcome than used in previous research. In the current study, the treatment effect in the model was adjusted for time, which allowed us to investigate whether or not the treatment effectiveness noted at the end of treatment was maintained or improved at 3 months follow-up, in addition to investigating the overall effect of the end of treatment and 3-month follow-up combined.

The results revealed several robust findings. As hypothesized, the CBT/MED group showed overall (combined outcome at post-treatment and follow-up) significantly greater reduction in all the primary outcomes of ADHD core symptoms and illness severity compared with TAU/MED group with a medium effect size for the independent raters on the K-SADS total and CGI and slightly less marked findings for the selfreported ADHD symptoms. A significant overall treatment effect was found for the secondary outcome of self-reported depression (small effect size) but, contrary to our hypothesis, this was not quite significant for anxiety and quality of life. As hypothesized, the treatment effect of primary outcomes was maintained at the 3-month follow-up.

Importantly, in contrast to the primary outcome measures, the three secondary measures (anxiety, depression and quality of life) showed a significant improvement over time, all with a medium effect size. This has not been investigated before. One interpretation is that the effect of the CBT treatment is more immediate with regard to the ADHD symptoms and illness severity, whereas symptoms of anxiety and depression and rating of quality of life improve more gradually over time, and may indeed continue to improve. The Emilsson et al. (2011) study did not include a measure of quality of life and the current study is the first study in this area to include one at follow-up. An alternative explanation is that the secondary measures effects are programme specific as the $R \mathcal{E} R 2 A D H D$ aims to reduce co-morbidity as well as primary symptoms. However, when comparing results from the present study with those reported by Emilsson et al. (2011), the effect sizes are generally smaller. This most probably reflects the use of a more sophisticated analysis that better controlled for confounders and thus provided more robust findings. A follow-up of secondary measures should be included in future studies, perhaps using a longer-term follow-up. The findings suggest that focusing exclusively on ADHD measures and symptom severity, like Safren et al. (2010), limits the conclusions that can be drawn about other health benefits and improved quality of life. This is particularly important in studies that include participants with a high base level of co-morbid problems. Hirvikoski et al. (2011) and Solanto et al. (2010) did collect data on co-morbid problems but they only included end-of-treatment data, which showed non-significant results. Ideally, treatment effectiveness should improve over time, or at least be maintained, as participants continue to practise the skills learned during therapy and gradually improve their level of competencies and day-to-day functioning. Further studies need to investigate the mechanisms that facilitate the maintenance and/or changes in outcome over time.

Just over half of the sample completed the group treatment. This is considerably lower than completion rates reported by forensic in-patient samples attending 
R\&R2 (Rees-Jones et al. 2012; Cin-Ying Yip et al. 2013; Young et al. 2013; Waugh et al. 2014; Jotangia et al. 2015). However, the attrition rate of patients attending community-led treatments has been reported to be high (e.g. Issakidis \& Andrews, 2004). Nevertheless, whilst the present study's completion rate is not particularly poor in comparison with other similar community studies, it means that many of the participants missed several of the sessions. In turn this may have adversely influenced the treatment effect, as participants who drop out are unlikely to reach optimal benefit from treatment. Ways for improving treatment completion in community samples need to be considered a priority in future studies.

A large number of participants (in both the CBT/ MED and TAU/MED groups) did not attend the sessions to complete their post-treatment assessments. A similar problem with missing data dropout was noted by Emilsson et al. (2011) and this was higher than that reported by Safren et al. (2010), Solanto et al. (2010) and Hirvikoski et al. (2011). It is not clear whether this is due to the nature of the participants, the severity of their symptoms, practical considerations such as the Icelandic climate (poor weather and travel conditions), or a reflection of the broader Icelandic community. For example, only $27 \%$ of patients attended their follow-up appointment in a large community study evaluating a substance misuse treatment in Reykjavik despite reminders by letter and a subsequent telephone call (Gudjonsson et al. 2004). Those patients who failed to attend their follow-up appointment were significantly younger than those who attended, were more antisocial in their personality, and had a higher level of trait anxiety. The current findings confirm the negative relationship between age and attendance at follow-up appointments, but not for ASP traits. Future studies should consider incorporating predictors of missing data into the linear mixed-model analysis in the event of group differences. This is particularly important when comparing participants who drop out of treatment versus those who complete all sessions (Everitt \& Pickles, 1999).

Strengths of the study were the use of independent raters who were blind to treatment condition and the consistency of these scores with those obtained from the participants' self-report. This suggests that the participants were not amplifying the benefits of treatment. The study's main limitation is the high dropout rate, which left us with a substantially reduced sample at follow-up. Second, in total there were 10 individual outcome measures (see Table 3), which increase the chance of a type I error although eight out of the 10 variables were significant at $\leq 0.001$, which reduces the likelihood this has occurred. However, the TAU/ MED group did not receive any additional control intervention, which may have inflated the treatment effects in the CBT/MED group.

The study adds substantially to knowledge by providing convincing evidence for the effectiveness of $R \mathcal{E} 2 A D H D$ and demonstrates that there are likely to be differential effects over time (end of treatment versus follow-up) with regard to primary ADHD outcomes versus co-morbid problems. Co-morbid problems are likely to take longer to show positive effect than the core ADHD symptoms. A possible explanation is that the disruptive behaviour commonly arising from ADHD symptoms increases the risk of depression due to the negative reactions of others to their behaviour (Roy, 2014). RER2ADHD treatment improves ADHD symptoms and disruptive behaviour, which over time are likely to reduce co-morbid symptoms due to a more positive reaction from peers, friends and family members. The current time difference found in treatment effects between core ADHD symptoms and co-morbid problems is likely to be related to the different developmental pathways of these conditions.

\section{Acknowledgements}

The authors wish to thank the patients for participating in the study. We acknowledge the contributions of Dr Sigurdur Pall Palsson for the randomization process, and Ragnhildur Georgsdottir and Emil Einarsson for their assistance in running the groups.

Support for the study was received from research grants awarded by RANNIS - the Icelandic Centre for Research (no. 080443022), the Landspitali Science Fund, and Janssen-Cilag, Iceland. No writing assistance was utilized in the writing of the manuscript. M.K. receives salary support from the National Institute for Health Research (NIHR) (Mental Health Biomedical Research Centre) at the South London and Maudsley NHS Foundation Trust and King's College London. The views expressed are those of the authors and not necessarily those of the NHS, the NIHR or the Department of Health.

\section{Declaration of Interest}

S.Y. has received consultancy fees, speaker's fees and/or travel honoraria from Janssen, Eli Lilly, Shire, Novartis and Flynn Pharma, and received research grants from Janssen, Eli Lilly and Shire. She is a consultant for the Cognitive Centre of Canada and coauthor of $R \mathcal{E} R 2 A D H D$. G.G. has received consultancy fees, speaker's fees and/or travel honoraria from Eli Lilly, Janssen and Shire. B.E. and G.B. have received travel honoraria from Janssen. The other authors have no competing interests. 


\section{References}

APA (1994). Diagnostic and Statistical Manual of Mental Disorders. American Psychiatric Association: Washington, DC.

Barkley R (1998). Attention Deficit/Hyperactivity Disorder: a Handbook for Diagnosis and Treatment. Guilford Press: New York.

Barkley RA, Murphy K, Fischer M (2008). ADHD in Adults: What the Science Says. Guilford Publications: New York.

Beck A, Steer R (1993). Beck Anxiety Inventory Manual. Harcourt Brace and Company: San Antonio.

Beck A, Ward C, Mendelson M (1961). Beck Depression Inventory (BDI). Archives of General Psychiatry 4, 561-571.

Beller EM, Gebski V, Keech AC (2002). Randomisation in clinical trials. Medical Journal of Australia 177, 565-567.

Bernardi S, Faraone SV, Cortese S, Kerridge BT, Pallanti S, Wang S, Blanco C (2012). The lifetime impact of attention deficit hyperactivity disorder: results from the National Epidemiologic Survey on Alcohol and Related Conditions (NESARC). Psychological Medicine 42, 875-887.

Biederman J, Faraone SV (2005). Attention-deficit hyperactivity disorder. Lancet 366, 237-248.

Bolea-Alamañac B, Nutt DJ, Adamou M, Asherson P, Bazire S, Coghill D, Heal D, Müller U, Nash J, Santosh P, Sayal K, Sonuga-Barke E, Young SJ (2014). Evidence-based guidelines for the pharmacological management of attention deficit hyperactivity disorder: update on recommendations from the British Association for Psychopharmacology. Journal of Psychopharmacology 28, 179-203.

Burckhardt CS, Anderson KL (2003). The Quality of Life Scale (QOLS): reliability, validity, and utilization. Health and Quality of Life Outcomes 1, 60.

Cin-Ying Yip V, Gudjonsson G, Perkins D, Doidge A, Hopkin G, Young S (2013). An evaluation of the R\&R2MHP cognitive skills program in high risk male offenders with severe mental illness. BMC Psychiatry 13, 267.

Emilsson B, Gudjonsson G, Sigurdsson JF, Einarsson E, Baldursson G, Olafsdottir H, Young S (2011). Cognitive behaviour therapy in medication-treated adults with ADHD and persistent symptoms: a randomized controlled trial. BMC Psychiatry 11, 116.

Everitt BS, Pickles A (1999). Statistical Aspects of the Design and Analysis of Clinical Trials. Imperial College Press: London.

Faul F, Erdfelder E, Lang AG, Buchner A (2007). G*Power 3: a flexible statistical power analysis program for the social, behavioral, and biomedical sciences. Behavior Research Methods 39, 175-191.

Flanagan JC (1978). A research approach to improving our quality of life. American Psychologist 33, 138-147.

Flanagan JC (1982). Measurement of quality of life: current state of the art. Archives of Physical Medicine and Rehabilitation 63, 56-59.

Friðriksdóttir N, Saevarsdóttir T, Halfdánardóttir $S$, Jónsdóttir A, Magnúsdóttir H, Olafsdóttir $\mathrm{K}$, Guðmundsdóttir G, Gunnarsdóttir S (2011). Family members of cancer patients: needs, quality of life and symptoms of anxiety and depression. Acta Oncologica 50, 252-258.
Gough HG (1960). Theory and measurement of socialization. Journal of Consulting and Clinical Psychology 24, 23-30.

Gudjonsson GH, Hannesdottir K, Agustsson T, Sigurdsson JF, Gudmundsdottir A, Pordardottir P, Tyrfingsson P, Petursson H (2004). Personality predictors of the failure of alcoholics to come for follow-up treatment. Personality and Individual Differences 37, 805-813.

Guldberg-Kjär T, Sehlin S, Johansson B (2013). ADHD symptoms across the lifespan in a population-based Swedish sample aged 65 to 80. International Psychogeriatrics 25, 667-675.

Hirvikoski T, Waaler E, Alfredsson J, Pihlgren C, Holmstrom A, Johnson A, Ruck J, Wiwe C, Bothen P, Nordstrom AL (2011). Reduced ADHD symptoms in adults with ADHD after structured skills training group: results from a randomized controlled trial. Behavior Research and Therapy 49, 175-185.

Issakidis C, Andrews G (2004). Pretreatment attrition and dropout in an outpatient clinic for anxiety disorders. Acta Psychiatrica Scandinavica 109, 426-433.

Jotangia A, Rees-Jones A, Gudjonsson GH, Young S (2015). A multi-site controlled trial of the R\&R2MHP cognitive skills program for mentally disordered female offenders. International Journal of Offender Therapy and Comparative Criminology 59, 539-559.

Kaufman J, Birmaher B, Brent D, Rao U, Ryan N (1996). Kiddie-SADS-Present and Lifetime Version (K-SADS-PL). Department of Psychiatry, University of Pittsburgh School of Medicine: Pittsburgh.

Magnússon P, Smári J, Sigurdardóttir D, Baldursson G, Sigmundsson J, Kristjánsson $\mathrm{K}$, Sigurdardóttir $\mathrm{S}$, Hreidarsson S, Sigurbjörnsdóttir S, Gudmundsson Óó (2006). Validity of self-report and informant rating scales of adult ADHD symptoms in comparison with a semistructured diagnostic interview. Journal of Attention Disorders 9, 494-503.

Mészáros Á, Czobor P, Bálint S, Komlósi S, Simon V, Bitter I (2009). Pharmacotherapy of adult attention deficit hyperactivity disorder (ADHD): a meta-analysis. International Journal of Neuropsychopharmacology 12, 1137-1147.

National Institute for Health and Clinical Excellence (2009). Attention Deficit Hyperactivity Disorder: Diagnosis and Management of ADHD in Children, Young People and Adults. NICE Clinical Guideline 72. NICE: London.

National Institute of Mental Health (1985). Clinical Global Impressions (CGI) Scale - NIMH. Psychopharmacological Bulletin 839-843.

Pliszka SR (1998). Comorbidity of attention-deficit/ hyperactivity disorder with psychiatric disorder: an overview. Journal of Clinical Psychiatry 59, 50-58.

Prince JB (2006). Pharmacotherapy of attention-deficit hyperactivity disorder in children and adolescents: update on new stimulant preparations, atomoxetine, and novel treatments. Child and Adolescent Psychiatric Clinics of North America 15, 13-50.

Rees-Jones A, Gudjonsson G, Young S (2012). A multi-site controlled trial of a cognitive skills programme for mentally disordered offenders. BMC Psychiatry 12, 44. 
Roy A (2014). Anxiety and disruptive hehaviour mediated pathways from attention-deficit/hyperactivity disorder to depression. Journal of Clinical Psychiatry 75, 108-113.

Safren SA (2006). Cognitive-behavioral approaches to ADHD treatment in adulthood. Journal of Clinical Psychiatry 67, 46-50.

Safren SA, Otto MW, Sprich S, Winett CL, Wilens TE, Biederman J (2005). Cognitive-behavioral therapy for ADHD in medication-treated adults with continued symptoms. Behaviour Research and Therapy 43, 831-842.

Safren SA, Sprich S, Mimiaga MJ, Surman C, Knouse L, Groves M, Otto MW (2010). Cognitive behavioral therapy vs relaxation with educational support for medicationtreated adults with ADHD and persistent symptoms: a randomized controlled trial. JAMA - Journal of the American Medical Association 304, 875-880.

Schulz KF, Grimes DA (2002). Allocation concealment in randomised trials: defending against deciphering. Lancet 359, 614-618.

Seixas M, Weiss M, Müller U (2012). Systematic review of national and international guidelines on attention-deficit hyperactivity disorder. Journal of Psychopharmacology 26, 753-765.

Shaw M, Hodgkins P, Caci H, Young S, Kahle J, Woods AG, Arnold LE (2012). A systematic review and analysis of long-term outcomes in attention deficit hyperactivity disorder: effects of treatment and non-treatment. $B M C$ Medicine 10, 99.

Sheehan DV, Lecrubier Y, Sheehan KH, Amorim P, Janavs J, Weiller E, Hergueta T, Baker R, Dunbar GC (1998).

The Mini-International Neuropsychiatric Interview (M.I.N. I.): the development and validation of a structured diagnostic psychiatric interview for DSM-IV and ICD-10. Journal of Clinical Psychiatry 59, 22-33.

Simon V, Czobor P, Balint S, Meszaros A, Bitter I (2009). Prevalence and correlates of adults attention-deficit hyperactivity disorder: meta-analysis. British Journal of Psychiatry 194, 204-211.

Sobanski E, Brüggemann D, Alm B, Kern S, Deschner M, Schubert T, Philipsen A, Rietschel M (2007). Psychiatric comorbidity and functional impairment in a clinically referred sample of adults with attention-deficit/ hyperactivity disorder (ADHD). European Archives of Psychiatry and Clinical Neuroscience 257, 371-377.

Solanto MV, Marks DJ, Wasserstein J, Mitchell K, Abikoff H, Alvir JM, Kofman MD (2010). Efficacy of meta-cognitive therapy for adult ADHD. American Journal of Psychiatry 167, 958-968.

Spencer TJ, Adler LA, Qiao M, Saylor KE, Brown TE, Holdnack JA, Schuh KJ, Trzepacz PT, Kelsey DK (2010).
Validation of the adult ADHD Investigator Symptom Rating Scale (AISRS). Journal of Attention Disorders 14, 57-68.

Stevenson CS, Whitmont S, Bornholt L, Livesey D, Stevenson RJ (2002). A cognitive remediation programme for adults with attention deficit hyperactivity disorder. Australian and New Zealand Journal of Psychiatry 36, 610-616.

Tong LS, Farrington D (2006). How effective is the "Reasoning and Rehabilitation" programme in reducing reoffending? A meta-analysis of evaluations in four countries. Psychology, Crime and Law 12, 3-24.

Waugh A, Gudjonsson GH, Rees-Jones A, Young S (2014). A feasibility study of the Reasoning and Rehabilitation Mental Health Programme (R\&R2MHP) in male offenders with intellectual disability. Criminal Behaviour and Mental Health 24, 222-224.

Weiss M, Murray C, Wasdell M, Greenfield B, Giles L, Hechtman L (2012). A randomized controlled trial of CBT therapy for adults with ADHD with and without medication. BMC Psychiatry 12, 30.

Wender PH (1998). Pharmacotherapy of attention-deficit/ hyperactivity disorder in adults. Journal of Clinical Psychiatry 59, 76-79.

White IR, Horton NI, Carpenter J, Pocock S (2011). Strategy for intention to treat analysis in randomised trials with missing outcome data. British Medical Journal 342, 910-912.

Wilens TE, Spencer TJ, Biederman J (2002). A review of the pharmacotherapy of adults with attention-deficit/ hyperactivity disorder. Journal of Attention Disorders 5, 189-202.

Willcutt EG (2012). The prevalence of DSM-IV attention-deficit/hyperactivity disorder: a meta-analytic review. Neurotherapeutics 9, 490-499.

Young S, Gudjonsson G (2006). ADHD symptomatology and it relationship with emotional, social and delinquency problems. Psychology, Crime and Law 12, 463-471.

Young S, Amarasinghe J (2010). Practitioner review: non-pharmacological treatments for ADHD: a lifespan approach. Journal of Child Psychology and Psychiatry and Allied Disciplines 51, 116-133.

Young S, Ross RR (2007). RER2 for ADHD Youths and Adults: a Prosocial Competence Training Program. Cognitive Centre of Canada: Ottawa.

Young S, Hopkin G, Perkins D, Farr C, Doidge A, Gudjonsson GH (2013). A controlled trial of a cognitive skills program for personality disordered offenders. Journal of Attention Disorders 17, 598-607. 